\title{
Vectorologie : orientations et progrès récents
}

\author{
Olivier Danos
}

\begin{abstract}
En dépit de l'importance des investissements et du foisonnement des projets de recherche, l'efficacité clinique de la thérapie génique n'est toujours pas démontrée. Dès 1995, la direction des NIH (national institutes of health) aux États-Unis s'en est inquiété et a désigné un comité chargé d'analyser la situation. Dans son rapport (http://www.nih.gov/news/panelrep.html), le comité co-présidé par Stuart Orkin et Arno Motulsky, reconnaît la validité des concepts qui fondent la thérapie génique mais souligne que les obstacles sont avant tout d'ordre fondamental. Les vecteurs de transfert de gène
\end{abstract}

y sont désignés comme l'élément limitant principal. Aucun d'entre eux, qu'il soit ou non dérivé de virus, ne présente l'efficacité et la sécurité requises pour une utilisation thérapeutique chez l'homme Ainsi, le comité Orkin-Motulski préconise de revenir à une recherche fondamentale et technologique de haut niveau afin de développer des outils performants. En outre, le rapport insiste sur la nécessité d'approfondir l'étude des mécanismes physiopathologiques des maladies et de développer des modèles animaux. a publication de ce rapport influence de manière décisive les orientations de la recherche en thérapie génique au cours des trois dernières années. A la bousculade médiatique des premiers temps, succède une période de profils plus bas, où l'on cherche à résoudre des problèmes difficiles. Des experts de tous bords tentent de comprendre les mécanismes qui rendent compte des limites connues pour chaque type de vecteurs. Le décloisonnement des recherches sur l'ensemble des vecteurs permet de dégager des thèmes transversaux. Parmi ceux-ci, citons le trafic intracellulaire, les phénomènes de recombinaison génétique, la régulation de l'expression des gènes, ou encore la réponse immunitaire au transfert de gène. Cet effort a déja conduit à des améliorations technologiques sensibles, en particulier dans le domaine des vecteurs viraux.

\section{Rétrovirus}

Les premiers vecteurs viraux pour le transfert de gène ont été dérivés d'un groupe de rétrovirus infectant la sou- ris (murine leukemia virus, MLV). Ces vecteurs peuvent être produits par des lignées cellulaires stables, et les conditions de leur préparation pour une utilisation clinique sont aujourd'hui bien établies (figure 1). Les meilleurs systèmes permettent d'obtenir des préparations concentrées (jusqu'à $10^{9}$ particules actives $/ \mathrm{ml}$ ), sans contamination par des virus compétents pour la réplication. Leur utilisation se limite essentiellement au transfert de gène $e x$ vivo, en particulier dans les cellules hématopoïétiques. L'application directe in vivo est d'une efficacité réduite car la cellule cible doit être en division pour que le transfert de gène ait lieu.

Les vecteurs dérivés d'une autre famille rétrovirale, les lentivirus, et en particulier du VIH, sont eux capables de transduire des cellules qui ne se divisent pas. Leur supériorité pour l'application directe in vivo (cerveau, rétine, muscle, foie) a été démontrée $[6,13]$. Il pourrait même être avantageux de les utiliser ex vivo, étant donné que les traitements mitogènes nécessaires à la transduction par un vecteur MLV, modifient sou- vent les propriétés des cellules, en particulier des cellules souches. Des systèmes de production stables et efficaces pour les vecteurs lentiviraux sont en cours d'élaboration [7]. Le problème majeur réside dans leur acceptabilité de ces vecteurs comme agents thérapeutiques. Bien que la possibilité d'obtenir la formation accidentelle de particules infectieuses du VIH lors de la préparation et de l'utilisation de ces vecteurs soit formellement exclue [3], il est clair que des critères sévères seront imposés pour la validation des vecteurs lentiviraux à usage thérapeutique.

\section{Adénovirus}

Les vecteurs adénoviraux peuvent être produits de manière relativement simple, à de très hautes concentrations de particules actives $\left(10^{12}\right.$ à $10^{13}$ particules $/ \mathrm{ml}$ ) (figure 2 ). Il demeurent les vecteurs les plus efficaces pour le transfert de gène in vivo, mais présentent de sérieux problèmes qui restreignent leur utilisation. L'infection par des adénovirus est commune dans les populations humaines. Il en résulte, chez la plu- 


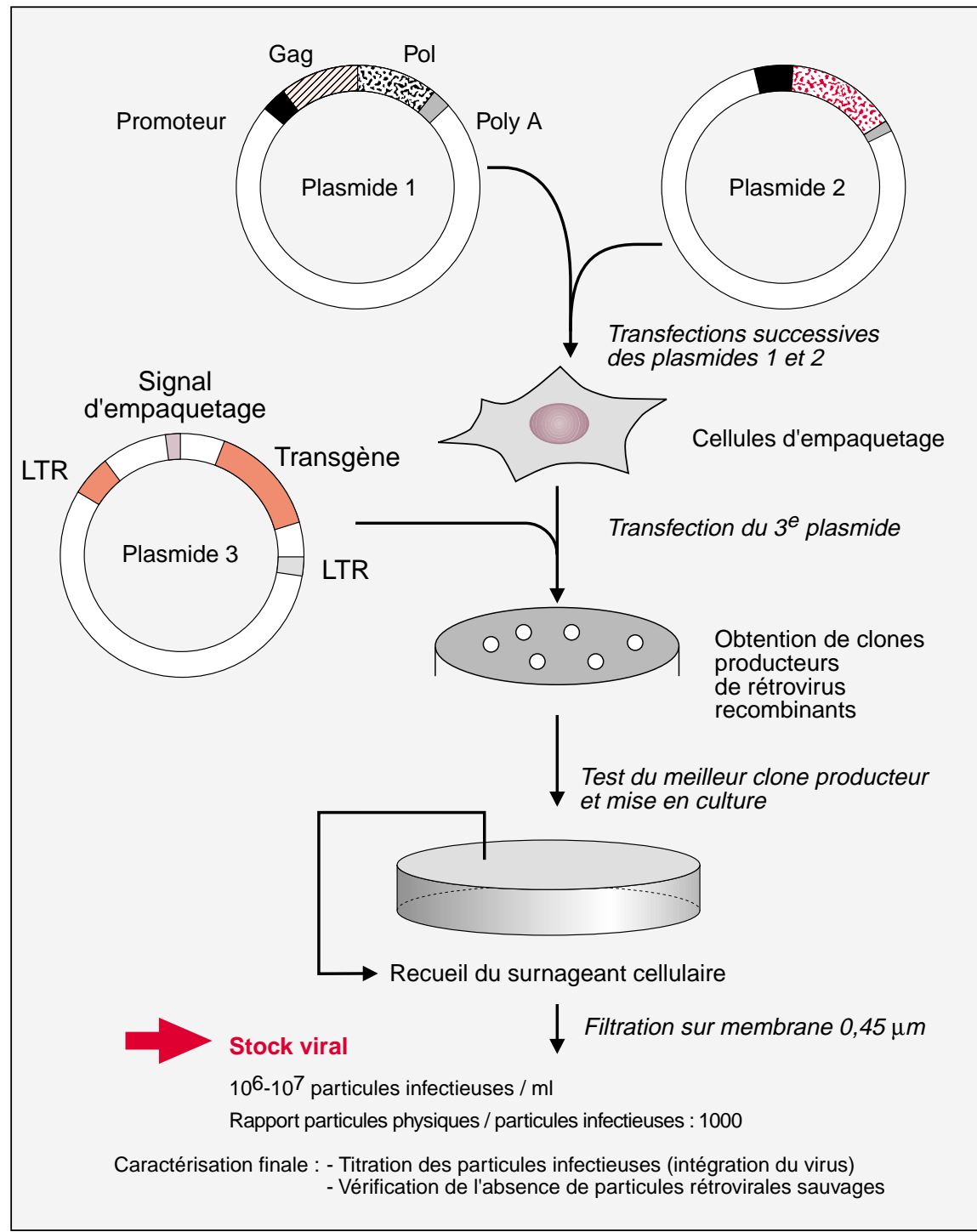

Figure 1. Préparation des vecteurs rétroviraux recombinants.

part des sujets, une immunité qui rend difficile l'utilisation des vecteurs. Il faut, de plus, considérer le risque qu'une infection naturelle mobilise un vecteur défectif et provoque sa dissémination. Outre l'immunité naturelle, on est confronté à celle provoquée par l'administration du vecteur lui-même, ou en réponse à l'expression résiduelle de protéines virales à partir des virus recombinants utilisés jusqu'à présent. Enfin, l'adénovirus n'est pas naturellement capable de maintenir son génome dans la cellule infectée, et en conséquence le gène transféré par un vecteur pourra être perdu au

La gageure actuelle consiste à proposer des solutions à ces problèmes sans perdre les avantages d'efficacité du système. Des vecteurs sont actuellement développés à partir d'adénovirus qui n'infectent pas naturellement l'homme, comme celui isolé chez le chien (CAV-2) [8]. On a d'autre part conçu des vecteurs minimaux dépourvus de toute séquence virale codante. Le mode de préparation de ces vecteurs minimaux dits gutless, qui peuvent véhiculer jusqu'à une trentaine de kilobases, est encore imparfait. Leur efficacité pour le transfert et le maintien d'un gène thérapeutique in vivo a cependant déjà été démontrée chez l'animal, dans le foie et dans le muscle [1, 2, 9]. Leur capacité étendue permet d'envisager d'y incorporer des systèmes permettant la pérennisation du transgène, tels que des séquences pouvant servir à sa réplication autonome ou à son intégration dans un chromosome de la cellule hôte. On arrive ainsi à l'idée de vecteurs gigognes, dans lesquels l'adénovirus servirait de plateforme de lancement pour un vecteur secondaire dont le rôle serait d'assurer le maintien des séquences transférées [4, 12].

\section{Virus associé à l'adénovirus}

Le virus associé à l'adénovirus (adenoassociated virus, AAV), un parvovirus humain non pathogène, a récemment retenu l'attention pour la construction de vecteurs (figure 3). Les propriétés du virus semblent conjuger les avantages des rétrovirus et de l'adénovirus. L'infection conduit dans certaines conditions à une modification génétique stable par intégration des séquences virales dans le génome de l'hôte, et ce en l'absence de division cellulaire. D'autre part, les virions sont extrêmement résistants et peuvent être produits à haute concentration (jusqu'à $10^{14}$ particules/ml [11]), ce qui les rend utilisables dans des approches de transfert de gène direct in vivo. Malheureusement, seule des séquences relativement courtes (moins de $5 \mathrm{~kb}$ ) peuvent être transportées par les vecteurs AAV. Même si nombre d'applications sont exclues d'emblée, la remarquable activité de ces vecteurs justifie largement l'intérêt qui leur est porté. Ce n'est que récemment que quelques équipes ont pu surmonter les difficultés liées à la préparation de ces vecteurs et les purifier en quantité suffisante pour pouvoir réaliser des essais sur l'animal. Les données publiées aujourd'hui illustrent la possibilité de transférer des gènes de manière permanente et efficace, en particulier dans le muscle, le cerveau, la rétine et le foie. Ces études ont été menées aussi bien sur des modèles murins que sur des gros animaux (chiens et singes). Des effets thérapeutiques ont été mesurés sur des modèles d'hémophilie B [5, 10]. Ces résultats sont remarquables 


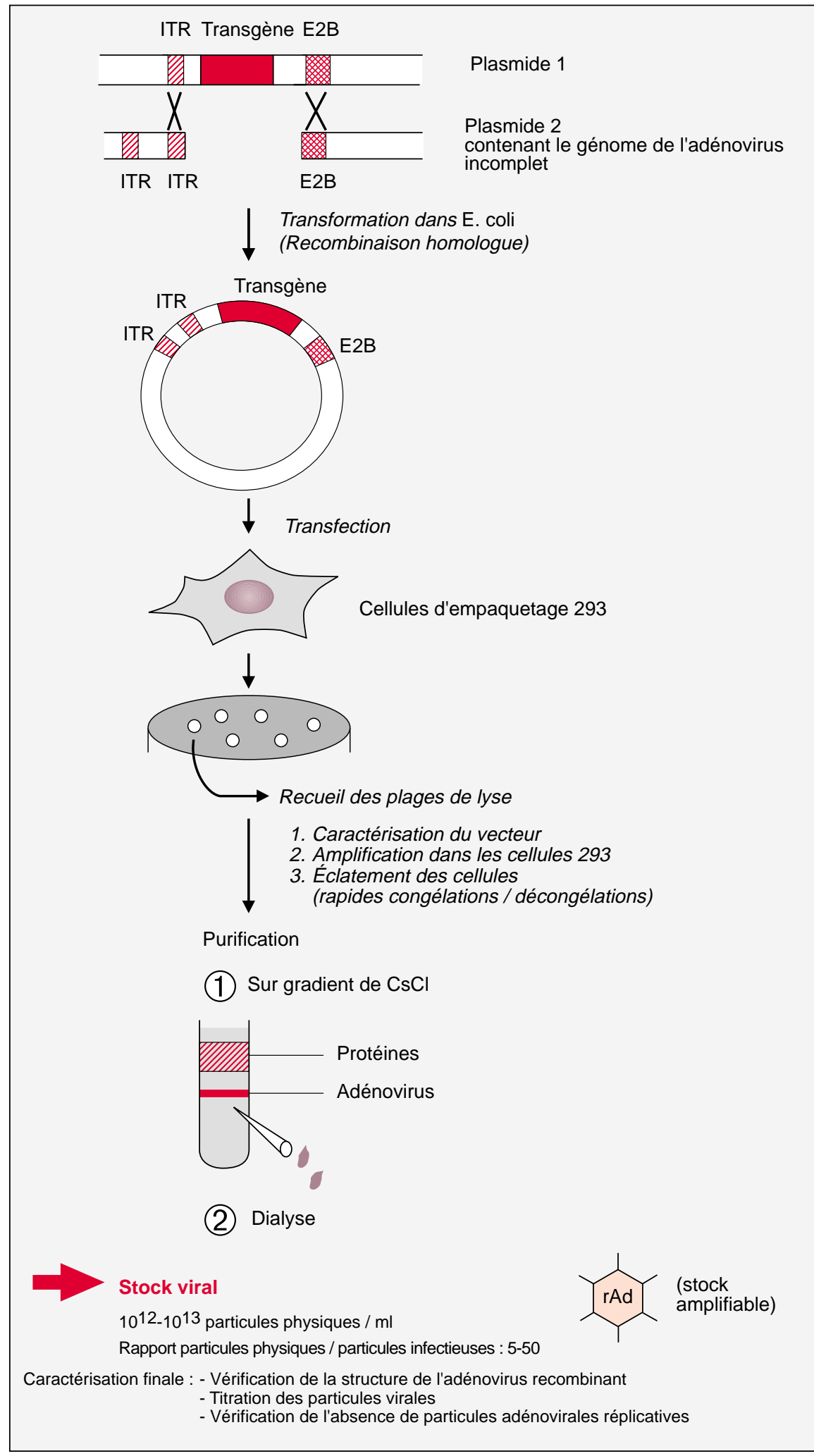

Figure 2. Préparation des vecteurs adénoviraux recombinants. parce qu'ils ont été obtenus à partir d'une administration unique de vecteur et que la réaction immune associée est limitée et transitoire. Ils conduisent aujourd'hui à la préparation de plusieurs essais cliniques. Les méthodes de préparation des vecteurs AAV restent cependant difficiles à transposer dans un environnement compatible avec la production de matériel à usage clinique. De nombreux efforts portent aujourd'hui sur l'adaptation de ce mode de production artisanal aux exigences des pratiques de fabrication de médicament. Notons enfin que bien des aspects du fonctionnement des vecteurs AAV demeurent obscurs. Il n'est par exemple pas clairement établi que le génome recombinant soit maintenu dans la cellule transduite par intégration dans le chromosome.

\section{Où trouver des vecteurs ?}

Ces progrès récents dans la technologie du transfert de gène, suscitent de nombreux projets chez les chercheurs, fondamentalistes et cliniciens. Mettre en place un savoir-faire de haut niveau en vectorologie demande cependant une expertise particulière et des investissements que tous ne sont pas prêts à supporter. Pour cette raison, on a vu se constituer, en particulier dans les centres universitaires américains, des unités de service dont la mission est de mettre à disposition des vecteurs. Ces facilités centrales constituent souvent l'élément de base des centres de thérapie génique qui ont éclos dans la plupart des centres hospitalouniversitaires. Certains centres proposent des vecteurs de qualité clinique, utilisables dans des essais de phase I et trois d'entre eux ont reçu des NIH le label National Gene Vector Laboratories (Universités de Pennsylvanie, d'Indiana et du Michigan, USA, http://www.iupui.edu/ iucc/ ngvl/). La proportion d'essais cliniques réalisés avec les vecteurs issus de ces centres reste faible, car leur capacité est limitée et la liste d'attente longue. En dehors de ces structures publiques, les chercheurs ont recours à des officines privées dont les prestations sont coûteuses. Les plus chanceux travaillent directe- 


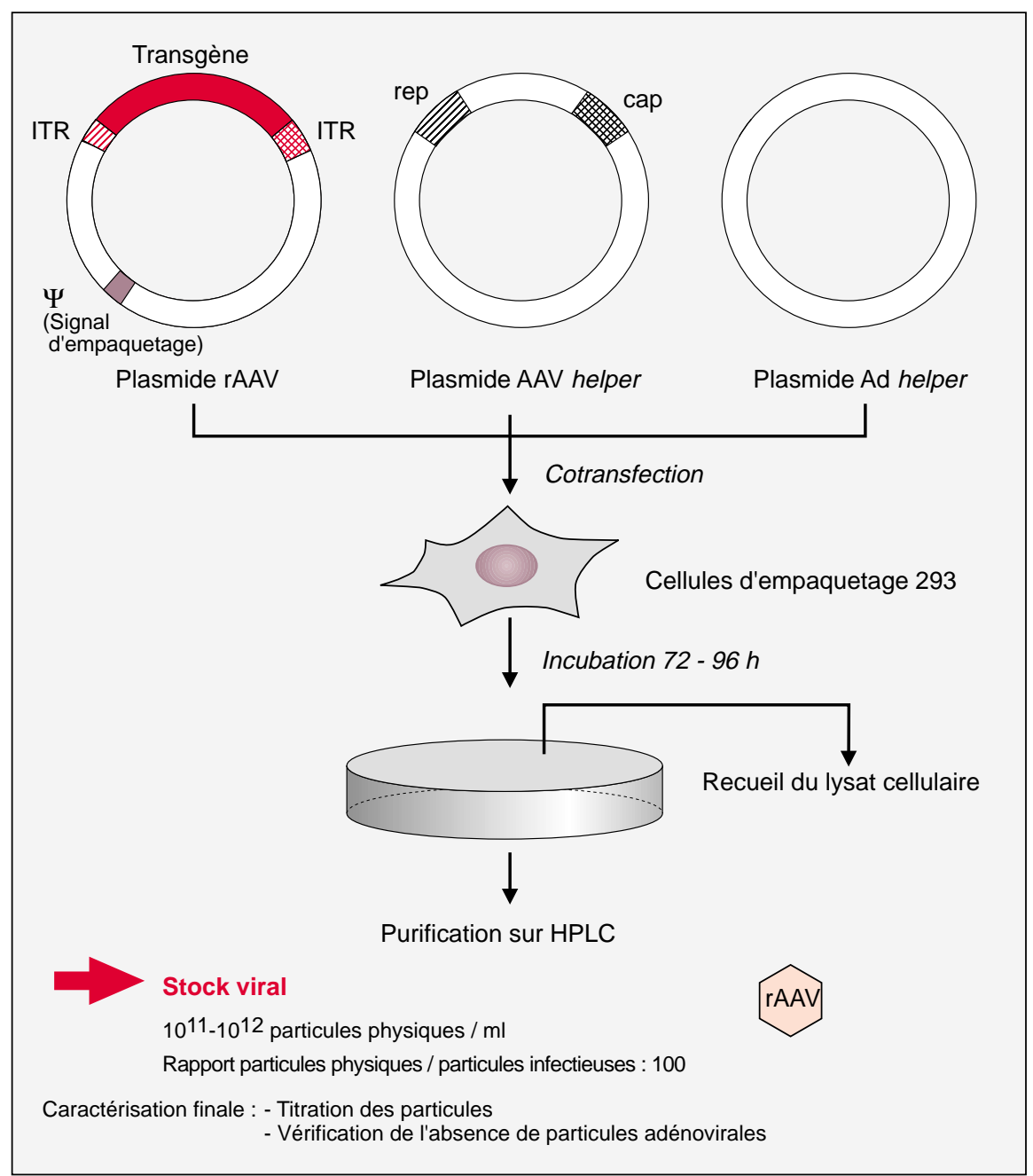

Figure 3. Préparation des vecteurs AAV recombinants.

ment avec une entreprise pharmaceutique directement intéressée par leur programme et qui prendra en charge la logistique de la production de vecteur.

En Europe aussi, une fois épuisées les ressources des collaborations académiques habituelles, les chercheurs ont recours pour la production de vecteurs à usage clinique, à des sociétés de service ou à des des industriels de la pharmacie. Quelques centres académiques sont apparus en Suède et en Belgique (Stockholm, Bruxelles), mais leur activité est très insuffisante pour absorber une demande grandissante. C'est pour répondre à cette situation que Généthon, le centre de recherches créé et financé par l'AFM (association française contre les myopathies), à Évry, réoriente depuis 1997 son activité en direction de la vectorologie. A côté d'une recherche de base menée en association avec le Cnrs, des procédés de production de vecteurs sont mis au point et appliqués dans une unité de service.

Certains parmi les plus fervents promoteurs de la thérapie génique ont dans un premier temps surestimé l'efficacité des outils qu'ils avaient entre les mains. L'excès d'enthousiasme n'a pas toujours servi leur cause, mais cette maladie infantile est aujourd'hui derrière nous. Une recherche de qualité est menée partout et les récentes démonstrations d'efficacité ne peuvent que convaincre les plus sceptiques. Les nouveaux principes de transfert de gène doivent être testés dans une large gamme de scénarios thérapeutiques et, pour cela, les vecteurs doivent être rendus aisément disponibles

\section{RÉFÉRENCES}

1. Burcin MM, Schiedner G, Kochanek S, Tsai SY, O’Malley BW. Adenovirus-mediated regulable target gene expression in vivo. Proc Natl Acad Sci USA 1999; 96 : 355-60.

2. Chen HH, Mack LM, Kelly R, Ontell M, Kochanek S, Clemens PR. Persistence in muscle of an adenoviral vector that lacks all virus genes. Proc Natl Acad Sci USA 1997; 94 : 1645-50.

3. Dull T, Zufferey R, Kelly M, et al. A thirdgeneration lentivirus vector with a conditional packaging system. J Virol 1998; 72: 8463-71.

4. Feng M, Jackson Jr WH, Goldman CK, et al. Stable in vivo gene transduction via a novel adenoviral/retroviral chimeric vector. Nat Biotechnol 1997; 15: 866-70.

5. Herzog RW, Yang EY, Couto LB, et al. Long term correction of canine hemophilia $B$ by gene transfer of blood coagulation factor IX mediated by Adeno-Associated Viral Vector. Nat Med 1999; 5: 56-63.

6. Kafri T, Blomer U, Peterson DA, Gage FH, Verma IM. Sustained expression of genes delivered directly into liver and muscle by lentiviral vectors. Nat Genet 1997; 17: 314-7.

7. Kafri T, Van Praag H, Ouyang L, Gage FH, Verma IM. A packaging cell line for lentivirus vectors. IVirol 1999; 73: 576-84.

8. Klonjkowski B, Gilardi-Hebenstreit P, Hadchouel J, et al. A recombinant E1-deleted canine adenovirus vector capable of transduction and expression of a transgene in human-derived cells and in vivo. Hum Gene Ther 1997; 8: 2103-15.

9. Morsy MA, Gu M, Motzel S, et al. An adenoviral vector deleted for all viral coding sequences results in enhanced safety and extended expression of a leptin transgene. Proc Natl Acad Sci USA 1998; 95 : 7866-71.

10. Snyder RO, Miao C, Meuse L, et al. Correction of hemophilia $\mathrm{B}$ in canine and murine models using recombinant adeno-associated viral vectors. Nat Med 1999; 5: 64-70.

11. Song S, Morgan M, Ellis T, et al. Sustained secretion of human alpha-1-antitrypsin from murine muscle transduced with adeno-associated-virus vectors. Proc Natl Acad Sci USA 1998; 95 : 14384-8.

12. Zhang L, Sankar U, Lampe DJ, Robertson HM, Graham FL. The Himarl mariner transposase cloned in a recombinant adenovirus vector is functional in mammalian cells. Nucleic Acids Res 1998; 26: 3687-93.

13. Zufferey R, Nagy D, Mandel RJ, Naldini L, Trono D. Multiply attenuated lentiviral vector achieves efficient gene delivery in vivo. Nat Biotechnol 1997; 15: 871-5.

\section{Olivier Danos}

Directeur de recherche au Cnrs, directeur scientifique, Cnrs URA 1923, Outils et Stratégies pour le transfert de gène thérapeutique, Généthon, 1, rue de l'Internationale, 91000 Évry, France. 


\section{LA PRODUCTION DE VECTEURS À GÉNÉTHON III}

La disponibilité de vecteurs de transfert de gène contrôlés, standardisés et de qualité optimale constitue pour les chercheurs et les cliniciens l'un des obstacles à l'exploration des nouvelles hypothèses thérapeutiques de la thérapie génique. La nouvelle mission du laboratoire Généthon (aujourd'hui rebaptisé Généthon III), est de lever cet obstacle en organisant la production en routine de vecteurs (AAV, MLV, HIV et $A d V)$. Les chercheurs français et étrangers utilisent déjà ce service qui propose aussi une assistance pour la conception des projets de transfert de gène et éventuellement une formation. Un système d'assurance qualité et de contrôle de qualité est mis en place, de façon à assurer la traçablité des produits. Ce point est essentiel si certains de ces vecteurs doivent parvenir aux stades de l'expérimentation clinique.

L'ambition de l'unité de production est de fournir des réactifs pour des projets de transfert de gène à différents niveaux de maturation. Les vecteurs pourront être utilisés dans les phases précoces des recherches en laboratoire, dans des études précliniques ou dans les premières phases de l'expérimentation clinique. Pour cela, il est nécessaire d'opérer dans des conditions de bonnes pratiques de laboratoire, voire de bonnes pratiques de fabrication. L'ouverture fin 1999 d'un atelier de production spécialement conçu à cet effet, permettra de réaliser cet objectif. On y assurera l'assemblage des constructions d'ADN recombinant, la constitution de banques cellulaires, l'isolement et la production des vecteurs, et enfin leur purification et caractérisation finale.

Doté de cette capacité de production de vecteurs, Généthon III s'insère dans un réseau auquel participent aussi le Centre de thérapie génique de l'Institut Paoli-Calmettes, à Marseille et le Laboratoire de thérapie génique du Centre Hospitalo-Universitaire de Nantes. Au niveau européen, Généthon III coordonne un projet de base de donnée et banque de vecteur (European Gene Vector Database and Repository). Dix partenaires (dont France, Belgique, Suède, Italie, Suisse, Angleterre et Israël) participent au projet qui doit réunir un catalogue de données sur les vecteurs disponibles (nature, structure, activité, disponibilité), et organiser la production et la distribution de certains d'entre eux.

\section{TIRÉS À PART}

O. Danos.

\section{Summary}

Vectorology: trends and progresses

Over the past few year, the field of gene therapy has placed a strong emphasis on vector research. This resulted from the outcome of the first series of clinical trials as well as from accumulated pre-clinical data. These collectively indicate that although the concept of gene therapy is valid, the tools we have in hand are far from being efficient enough. Three successive levels of problem exist. First, much fundamental investigation on the mechanism of gene transfer is needed and novel types of vectors have to be engineered. Second, the vector research must be linked to the development of optimised procedure for the production of reagents to be used in clinical trials. Finally, production facilities accessible to the scientific community must be organised. Here, we briefly describe the most significant progresses in viral vectors development (retroviruses, adenoviruses and adeno-associated viruses), and the current efforts designed to facilitate access to high quality vectors to investigators with a gene therapy project. 